\title{
The Piezo1 Mechanosensitive Ion Channel Promotes Melanoma Malignant Progression by Activating PI3K-AKT Signaling
}

simei zhang

Xian Jiaotong University: Xi'an Jiaotong University

shuang cao

Charite Universitatsmedizin Berlin Campus Charite Mitte: Charite Universitatsmedizin Berlin

mengyuan gong

Xian Jiaotong University: Xi'an Jiaotong University

wunai zhang

Xian Jiaotong University: Xi'an Jiaotong University

weifan zhang

Xian Jiaotong University: Xi'an Jiaotong University

zeen zhu

Xian Jiaotong University: Xi'an Jiaotong University

shuai wu

Xian Jiaotong University: Xi'an Jiaotong University

yue shi

Xian Jiaotong University: Xi'an Jiaotong University

lin wang

Xian Jiaotong University: Xi'an Jiaotong University

yangyang yue

Xian Jiaotong University: Xi'an Jiaotong University

weikun qian

Xian Jiaotong University: Xi'an Jiaotong University

qingyong ma

Xian Jiaotong University: Xi'an Jiaotong University

shengpeng wang

Xian Jiaotong University: Xi'an Jiaotong University

zheng wang ( $\nabla$ zheng.wang11@mail.xjtu.edu.cn)

First Affiliated Hospital of Xi'an Jiaotong University

Research 
Keywords: Piezo1, proliferation, metastasis, melanoma, PI3K-AKT signaling

Posted Date: July 6th, 2021

DOI: https://doi.org/10.21203/rs.3.rs-638409/v1

License: (c) (1) This work is licensed under a Creative Commons Attribution 4.0 International License. Read Full License 


\section{Abstract}

Background: Melanoma is a highly aggressive cancer that can metastasize at early stage. The mechanosensitive ion channel Piezo1 plays a crucial role in embryonic development, tumour growth, migration, invasion and vascularization. The aim of this study was to clarify the role of Piezo1 and its potential mechanism in regulating the malignant phenotypes of melanoma.

Methods: The expression of Piezo1 in melanoma was analysed using quantitative real-time PCR and public databases. The effect of Piezo1 on cell viability was examined using a cell counting kit-8 assay. Cell invasion and migration ability were assessed using wound healing assays, transwell assays, transendothelial migration assays and a tail vein cancer metastasis model in vivo. Bioinformatics and western blot assayses were used to explore the effect of Pieoz1 on P13K/AKT signalling.

Results: Piezo1 was upregulated in melanoma and was positively associated with poor survival. Piezo1 knockdown significantly weakened the intracellular calcium signal significantly and inhibited the viability of melanoma cells. Furthermore, Piezo1 knockdown inhibited the invasion and metastasis ability in vitro and in vivo by inducing the expression of cell cycle, invasion and metastasis related genes. To clarify the possible mechanism, it seems that Piezo1 activates the PI3K-AKT signalling to maintain malignant phenotypes of melanoma.

Conclusion: Piezo1 acts as an oncogene in melanoma cells and provides a novel candidate for melanoma diagnosis and treatment.

\section{Background}

Malignant melanoma is one of the most aggressive cancers worldwide, with a rising incidence rate. In 2019 , an estimated 96,480 new melanoma cases and 7230 melanoma deaths were occurred in the United States[1]. Melanoma is a highly aggressive tumor and exhibit early metastasis [2], which is one of the reasons for poor clinical outcomes [3]. Therefore, there is an urgent need to unravel the molecular mechanisms of the malignant progression of melanoma, and provide a prospective therapeutic strategy.

With the knowledge of metastasis, we focused on the mechanosensitive channel proteins. Piezo1, an important mechanosensitive channel proteins, was first identified in2010 [4]. The shape of the Piezo1 ion channel is a trimeric, three-bladed propeller structure consisting of three peripheral blade-like structures and a central cap-like structure $[5,6]$. The Piezo1 ion channel protein is capable of sensing mechanical stimuli such as pressure, tension and fluid shear. In the activated state, it promotes calcium influx into cells[7]. As a second messenger, intracellular $\mathrm{Ca}^{2+}$ can regulate cell migration, invasion, proliferation and gene transcription. In addition, Piezo1 plays a crucial role in embryonic development [8].The role of Piezo1 as an ion channel protein capable of sensing mechanical stimuli has been gradually investigated in tumours $[9,10]$. However, it is unknown how Piezo1 influences the malignant behavior of melanoma. 
In the present study, we are the first to show that Piezo1 is abnormally expressed in melanoma, which accelerates the malignant progression. We further found that Piezo1 regulates invasion, metastasis and cell cycle related genes and activates PI3K/AKT signalling to control the viability, metastasis, invasion, and transendothelial migration in vitro and in vivo.

\section{Methods}

\section{Cells and Reagents}

The human A375 melanoma cell line, mouse B16 melanoma cells, and human umbilical vein vascular endothelial cell (HUVEC) were purchased from the American Type Culture Collection (ATCC, US). All cell lines were cultured in Dulbecco's modified Eagle's medium (DMEM; Gibco; Thermo Fisher Scientific, USA) with $10 \%$ foetal bovine serum at $37^{\circ} \mathrm{C}$ aired with $5 \% \mathrm{CO}_{2}$. The following antibodies were used in this study at the indicated dilutions for western blot (WB) and immunofluorescence (IF): Piezo1(Ca-15939-1-AP, 1:1000 for WB), MMP2(10373-2-AP,1:1000 for WB), and beta-actin (66009-1-1g, 1:2000 for WB) were purchased from Proteintech. Ki-67(9129, 1:400 for IF), AKT (4691, 1:1000 for WB), p-AKT (Thr308, 13038, 1:1000 for WB), E-cadherin (3195, 1:1000 for WB), N-cadherin (13116, 1:1000 for WB), and vimentin (5741, 1:1000 for WB) were purchased from CST (Cell signaling Technology, Inc). MMP9 (ab38898, 1:1000 for WB) was purchased from Abcam.

\section{siRNA /shRNA and lentiviral transduction}

siRNAs were purchased from Qiagen. At cell density of $1-2 \times 10^{4} / \mathrm{cm}^{2}, 50 \mathrm{pmol}$ of siRNA was mixed gently with RNAiMAX in $100 \mu \mathrm{l}$ of Opti-MEM, incubated for $30 \mathrm{~min}$ at room temperature and added to 1.5 $\mathrm{ml}$ of cell culture medium (DMEM). Cells were incubated with the complexes for $8-12 \mathrm{~h}$ at $37^{\circ} \mathrm{C}$ in a $\mathrm{CO}_{2}$ incubator and thereafter, the medium was replaced with complete medium (DMEM plus $10 \% \mathrm{FBS}$ ). For RNA and protein experiment, cells were harvested $24 \mathrm{~h}$ and $48 \mathrm{~h}$ posttransfection, respectively. The siRNA target sequences directed against RNAs encoding Piezo1 were 5'-CACCGGCATCTACGTCAAATA- 3' and 5'TCGGCGCTTGCTAGAACTTCA-3'. Piezo1 knockdown mouse melanoma cells were named siNC and siPiezo1.

Piezo1 knockdown lentivirus and scramble control were purchased from GeneCopoeia (Guangzhou, China). The target sequence for Piezo1 shRNA was GGTCTACAAGATTGTCTACAT, and the negative control sequence was GCTTCGCGCCGTAGTCTTA. Transfection was carried out using lentiviral particles and polybrene according to the manufacturer's protocol. Cells were selected with puromycin $(4 \mu \mathrm{g} / \mathrm{ml})$ for 10 days. Stable Piezo1 knockdown human melanoma cells were named shNC and shPiezo1. The effect of gene silencing was analysed by real-time PCR and western blot.

\section{Determination of intracellular $\left[\mathrm{Ca}^{2+}\right]$}

To determine of the intracellular $\mathrm{Ca}^{2+}$ concentration, cells were placed in 96-well microplates for fluorescence-based assays (Invitrogen) and loaded with Fluo-4 AM (Molecular Probes, Life Technologies). 
Live-cell images were acquired with an Olympus IX81 microscope. Fluorescence intensity was measured with a FlexStation 3 (Molecular Devices).

\section{Immunofluoresence}

Cells plated on coverslips were fixed with $4 \%$ paraformaldehyde for 30 min, permeabilized with $0.2 \%$ Triton X-100 for 10 min, and blocked with 5\% bovine serum albumin (Sigma-Aldrich, Germany) for 1 hour. Then the cells were incubated overnight with a primary antibody of Ki67. Next, the samples were extensively washed with PBS buffer, incubated with fluorescein isothiocyanate-conjugated AffiniPure goat anti-rabbit IgG secondary antibody (dilution, 1:200; cat, EF00002, SPARK JADE) for 60 min and stained with DAPI (cat, D1306,1:5000, Invitrogen) for 5 min in the dark. Laser scanning confocal microscopy (Nikon A1R/A1) was used to observe the samples.

\section{Cell viability assay}

A375 and B16 cells were seeded in 96-well plates at 1000 cells and $200 \mu$ per well, respectively, and incubated overnight in 10\% FBS medium. After 24 h, 48 h, 72 h, and 96 h, 20 ll of CCK-8 (Dojindo Molecular Technologies, Inc., Kumamoto, Japan) was added to $180 \mu$ l of completed medium per well, and the absorbance was determined at $490 \mathrm{~nm}$. The data are the result of three independent experiments.

\section{Wound healing assay and transwell assay}

The A375 and B16 melanoma cell lines were seeded on 6-well plates, and when the cells reached 100\% confluenceusing, a $200 \mu \mathrm{l}$ pipette tip was used to scratch the cells to create artificial wounds. Wound healing was observed by inverted microscopy after $24 \mathrm{~h}$.

Transwell invasion assays were performed in 24-well plates. Transwell chambers (pore size, $8.0 \mu \mathrm{m}$; Millipore, Billerica, USA) were coated with Matrigel (BD Bioscience, Oxford, UK). A375 and B16 (300 $\mu$ l, $5 \times 10^{4}$ cells per well ) suspended in DMEM containing free-FBS were seeded in the top chamber, and 600 $\mu \mathrm{l}$ medium containing 10\% FBS was placed in the lower chamber. After $24 \mathrm{~h}$, cells that to penetrate to the lower surface of the chamber were fixed with paraformaldehyde for $20 \mathrm{~min}$ and then stained with $0.1 \%$ crystal violet for $10 \mathrm{~min}$. Cell migration and invasion were determined by counting the stained cells under a light microscope in 5 randomly selected fields.

\section{Transendothelial migration assay}

Tumour cell transendothelial migration assays were performed using transwell chambers that were coated with Matrigel 8 hours in advance. HUVECs were seeded in the top chamber until they reached $100 \%$ confluence, and A375 and B16 (300 $\mu \mathrm{l}, 6 \times 10^{4}$ cells per well ) suspended in FBS-free DMEM were seeded in the top chamber, and $800 \mu$ medium containing 10\% FBS was placed in the lower chamber. After 12 hours, the upper layer of HUVECs and the tumour cells that had not crossed the endothelium were removed using a cotton swab. Then $50 \mu \mathrm{l}$ and $250 \mu \mathrm{l}$ of $1 \times$ PBS were added to the upper and lower layers, respectively, and the tumour cells were counted under fluorescence microscopy. 


\section{Quantitative Real-time PCR assay (qRT-PCR)}

Total RNA was isolated with RNAfast 200 reagents (Fastagen Biotechnology, Shanghai, China). After quantitation by absorbance at $260 \mathrm{~nm}, 1000 \mathrm{ng}$ of the total RNA was reverse transcribed by PrimeScript RT Master Mix (Takara Bio, Dalian, China). Quantitative PCR was carried out using SYBR-Green PCR Master Mix (Takara Bio, Dalian, China) with the following specific primers: Piezo1(human), F: cgtcttcgtggagcagatg, R: gcccttgacggtgcatac; Piezo1(mouse), F: ggaaaagagctccgacacac-3 R: ccaggacttccccacctatt; 18S, F: cagccacccgagattgagca, R: tagtagcgacgggeggtgtg.

\section{Western blot analysis}

Cells were lysed in RIPA buffer ( $50 \mathrm{mM}$ Tris, pH 8.0,150 mM NaCl, 0.1\% SDS, $1 \%$ NP40 and 0.5\% sodium deoxycholate) supplemented with protease inhibitor (1\% inhibitors cocktail and $1 \mathrm{mM} \mathrm{PMSF}$ ) (Roche Applied Science, Germany). Lysates were centrifuged at $12000 \mathrm{~g}$ for $15 \mathrm{~min}$ at $4^{\circ} \mathrm{C}$, subjected to $8 \% \mathrm{SDS}$ PAGE and transferred to polyvinylidene fluoride (PVDF) membranes. Membranes were incubated with the indicated primary antibody at $4^{\circ} \mathrm{C}$ overnight and were developed using the $\mathrm{ECL}$ chemiluminescent detection system (BioRad, USA). The experiment was repeated three times.

\section{In vivo cell extravasation assay}

Piezo1-silenced B16 melanoma cells were suspended in $1 \times$ PBS and stained with $1 \mu \mathrm{g} / \mathrm{ml} \mathrm{CFSE}($ Invitrogen, US) for $15 \mathrm{~min}$ at $37^{\circ} \mathrm{C}$. Cells were then washed with PBS, suspended in complete medium and incubated for $30 \mathrm{~min}$ at $37^{\circ} \mathrm{C}$. Then, cells $\left(1.5 \times 10^{5}, 100 \mu \mathrm{l}\right)$ were suspended in PBS and introduced into the mice by tail-vein injection. After 6 hours, mice were sacrificed and lung tissues were analysed by immunofluorescence (IF) [11]to label the vascular endothelial cells of the lungs and observe the tumour cells inside and outside the blood vessels.

\section{In vivo metastasis tumour model}

The tail-vein injection metastasis model was generated as described in previous studies. Wild-type (WT) (C57/BL6J) mice (8 weeks) were used according to protocols approved by the Ethics Committee of Xi'an Jiaotong University. Piezo1-silenced B16 melanoma cells with luciferase markers $\left(1.5 \times 10^{5}\right.$ cells, $\left.100 \mu l\right)$ were suspended in serum-PBS and introduced into the circulation of mice by tail-vein injection. After 6 weeks, $150 \mathrm{mg} / \mathrm{kg}$ D-luciferin substrate (Biosynth, Naperville, IL, USA) in PBS was injected into the abdominal cavity. Fifteen to 20 min later, bioluminescence imaging (BLI) was performed to detect distant metastases in the lung and other organs after the mice were anesthetized. The mice were then sacrificed, the lungs were lavaged with PBS to clear them, and the number of melanoma metastases was counted under a microscope (Invitrogen, USA).

\section{Bioinformatics and Statistical analyses}

The GSE46517 dataset was used to analyse the expression of Piezo1 in normal skin, primary melanoma and metastatic melanoma. The overall survival-related Piezo1 expression was analysed from the Human Protein Atlas website (https://www.proteinatlas.org/). The GEPIA online bioinformatics analysis websites 
(http://gepia.cancer-pku.cn/) was used to analyse the relationship between the levels of Piezo1 target genes and AKT pathway molecules. The data were analysed and graphed using GraphPad Prism version 7.0 software (GraphPad Software, USA). Comparisons between multiple groups ( $\geq 3$ ) were performed using one-way ANOVA. Other statistical analyses were performed using two-tailed Student's t-tests. P values $<0.05$ were considered statistically significant.

\section{Result}

Piezo1 promotes melanoma cell viability

We analysed the copy number of Piezo1 in several tumour cells, and Piezo1was widely expressed in multiple tumour cell lines (Fig. S1A). Bioinformatics analysis was also used to explore the expression level of Piezo1 in melanoma. We found that the Piezo1 mRNA expression level was upregulated in metastatic melanoma and primary melanoma compared with normal skin tissues in TCGA (Fig. S1B). Human Protein Atlas website datasets showed that the upregulation of Piezo1 was correlated with the shorter overall survival of melanoma patients (Fig. S1C). To investigate the role of Piezo1 in melanoma cells, the stable A375 cell line was established successfully with Piezo1 shRNA, Piezo1 knockdown of B16 was performed with (mouse)-Piezo1 siRNA, and the efficiency of knockdown was determined by qRTPCR and western blot analysis (Fig. 1a, b). Furthermore, we selected two cell lines with significant knockdown effects for subsequent experiments. When control cells were stimulated with Yoda1 (an agonist of Piezo1, $10 \mu \mathrm{M})$, intracellular $\left[\mathrm{Ca}^{2+}\right]_{i}$ recordings showed a transient increase in the free cytosolic $\mathrm{Ca}^{2+}$ concentration. However, in the Piezo1 knockdown group, the intracellular calcium signal weakened significantly, and enhancement of the signal was very slow (Fig. 1c). The results of this experiment suggest that the Piezo1 ion channel protein is functional in melanoma cells.

To determine whether Piezo1 affects melanoma cell proliferation, a CCK-8 assay was applied to continuously monitor changes in cell viability at 0, 24, 48, 72, and $96 \mathrm{~h}$. As shown in Fig. 1d, knockdown of Piezo1 suppressed the viability of melanoma cells. DNA replication activity is usually considered to represent proliferation capacity and was determined by cell staining with Ki67 antibody. There was reduced Ki67 staining after Piezo1 knockdown (Fig. 1e). Taken together, these results indicated that Piezo1 loss may suppress melanoma cell viability.

Knockdown of Piezo1 inhibits the migration, invasion and transendothelial migration of melanoma cells

Previous studies have clarified that intracellular calcium signalling is also associated with reorganization of the cytoskeleton such as F-actin, stress fiber and the formation of lamellipodia, which play a vital role in regulating tumour invasion and metastasis $[3,12]$. Our result confirmed that activation of the mechanically sensitive Piezo1 ion channel promotes inward calcium flow. Therefore, the potential role of Piezo1 in the migration and invasion capacity of melanoma cells was explored. The results suggested that efficient knockdown of Piezo1 significantly impaired the wound healing rate and invasiveness ability compared with the control group (Fig. 2a-c). Aaaitionally, a transendothelial migration assay was used to 
verify the role of Piezo1 in transendothelial migration of tumour cells. The results showed that the migration ability of Piezo1 knockdown cells crossing the HUVACs was significantly reduced (Fig. 2d).

Piezo1 inhibition blocks invasion, metastasis and cell cycle related genes in vitro

The data indicated that Piezo1 inhibition suppressed melanoma cell viability. In addition, expression of the cell cycle-related CDK2 and cyclinD1 genes decreased after Piezo1 knockdown. However, P21 and PTEN, classical tumour suppressors were increased (Fig. 3a). Numerous studies have suggested that epithelial-to-mesenchymal transition (EMT) contributes to early-stage dissemination of cancer cells and is pivotal for invasion and metastasis of melanoma $[13,14]$. In addition, matrix metalloproteinases (MMP2, MMP9) play vital roles in tissue remolding and cancer metastasis [15]. The results showed that mesenchymal-related gene (N-cadherin and E-cadherin), invasion-and metastasis-related gene (MMP2 and MMP9) expression in melanoma cells were significantly decreased after Piezo1 loss (Fig. 3b). Taken together, our observations indicated that Piezo1 is critical in malignant tumour events, including invasion and metastasis.

Piezo1 inhibition inactivates the PI3k/AKT pathway

Evidence indicates that, PI3K/AKT signalling is frequently activated in most malignant cancers, and regulates tumour cell proliferation, adhesion, survival, migration and invasion [2, 16, 17]. In addition, activation of PI3K/AKT induces tumour cell migration and invasion through degradation of the MMPsmediated matrix [18]. We analysed several possible candidates that respond to mechanical biosignalling pathways by the online bioinformatics GEPIA websites and found that Piezo1 is associated with AKT pathway molecules (Fig. 2a). Therefore, we treated melanoma cells with Yoda1 (a Piezo1 activator) at various concentrations (Supplementary Fig. 2) and selected $10 \mu \mathrm{M}$ as the most effective concentration. The results indicated that phosphorylation of AKT was decreased in melanoma cells after knockdown of Piezo1 (Fig. 3c). Furthermore, we treated melanoma cells with Yoda1 to investigate which pathways were associated with the activation of Piezo1. AKT pathway was activated after the administration of Yoda1 (Fig. 3d-e). The above results indicate that the phosphorylation of AKT requires the activation of Piezo1. Taken together, these data revealed that the level of activation of the mechanical ion channel Piezo1 increased the activation of the PI3K/AKT pathway.

Piezo1 promotes the melanoma cell proliferation, metastasis and invasion via the PI3K/AKT signaling pathway

To further clarify whether the function of Piezo1 in regulating the malignant progression of melanoma was dependent on the PI3K/AKT pathway, we treated wild-type A375 cells with the PI3K inhibitor LY294002, the Piezo1 activator Yoda1 and both. Blocking PI3K not only inhibited migration, invasion and transendothelial migration in control cells but also significantly reduced the migration, invasion and transendothelial migration induced by Piezo1 activation with Yoda1 (Fig. 4a-d). Moreover, as shown in Fig. 4e-f, p-AKT, MMP2, and N- cadherin protein levels were increased by the Piezo1 activator Yoda1, while LY294002 treatment reversed the expression of p-AKT but also MMP2, and N-cadherin. These results 
collectively indicated that the oncogenic property of Piezo1 was mediated by activation of the PI3K/AKT signalling pathway.

Knockdown of Piezo1 attenuated melanoma metastasis and transendothelial migration in vivo

Based on the results obtained from in vitro experiments, we verified the role of Piezo1 in vascular extravasation of tumour cells in vivo using the lung metastasis mouse model. By injecting B16 cells (siPiezo1and siNC fluorescently labelled) into mice and collecting lung tissue for frozen sectioning after 6 hours, we surprisingly found that the tumor cells that the number of tumour cells that penetrated the blood vessels was 50\% lower in the Piezo1-silenced group than in the control group. (Fig. 5a).

The tumour metastasis-promoting role of Piezo1 in melanoma was verified in vivo. We established a lung metastasis mouse model using the tail-vein injection model of B16 cell-lines. Two weeks later, the fluorescence signals of tumour cells in mice were tracked using a bioluminescence imaging system, and the results showed that there were less tumour signals in Piezo1-silenced tumour cells. Since melanoma appears as black spots on lung tissue, the mice were sacrificed, and lung tissue was collected. The results showed that the lungs of the shPiezo1 mice had only a few scattered black spots, while the control mice had evenly spaced spots (Fig. 5b, c). These data suggest that the Piezo1 channel may promote the metastasis of melanoma in vivo. Therefore, Piezo1 plays an important role in the transvascular migration of tumour cells and provides a basis for distant metastasis of the tumour.

\section{Discussion}

Although the incidence is low, malignant melanoma is highly invasive and difficult to treat because of rapid malignant progression at an early stage. Therefore, the poor prognosis of melanoma has not improved $[19,20]$. Hence, it is important to investigate the mechanisms that cause the early malignant progression of melanoma. Here, we first demonstrate a previously unrecognized role of the Piezo1 mechanosensitive ion channel in promoting tumour aggression in melanoma.

Alteration of mechanical properties is a physical hallmark of solid tumours, including melanoma. Increased tissue stiffness actively promotes malignant progression by affecting proliferation, migration, invasion, drug resistance, and growth-promoting signalling [21, 22]. Studies shown that Piezo1 mediates breast cancer cell migration by regulating cell stiffness, contractility, and adhesiveness [10, 23]. In addition, Piezo1 has oncogenic effects in gastric cancer cells, including effects on cell migration, invasion and proliferation [24]. In gliomas, Piezo1 channels interact with integrin-FAK signalling and further promote tumour cell proliferation and tissue stiffness. However, it remains unclear how Piezo1 regulates malignant progression in melanoma.

In our study, we expected to identify the role of Piezo1 in melanoma in vitro and in vivo and its underlying mechanisms. We found that Piezo1 was widely overexpressed in melanoma cells, and promoted proliferation, invasion, metastasis, transendothelial migration and distant metastasis in vitro and in vivo. Our results showed that knockdown of Piezo1 suppressed the viability of melanoma cells and decreased 
the expression of Cyclin D1 and CDK2. Cyclin D1 and CDK2 are the key effectors in regulating cell cycle transitions. This result indicated that inhibition of Piezo1 expression caused cell cycle arrest, which is consistent with a previous study [25]. Evidence suggests that fluid shear stress targets tumour bone morphogenetic and integrin proteins, which accelerates tumour cell cycle arrest [26]. However, the relationship between fluid shear stress and Piezo1 has not been elucidated thus far. Our results indicated that activation of Piezo1 increase in the free cytosolic $\mathrm{Ca}^{2+}$ concentration. Intracellular $\mathrm{Ca}^{2+}$, as a second messenger, mediates various cellular processes, including gene transcription, cell migration and invasion [27]. Moreover, intracellular $\mathrm{Ca}^{2+}$ regulates the $\mathrm{G} 1$ to $\mathrm{G} 2$ transition, especially the $\mathrm{G} 1$ transition [28]. In melanoma, intracellular $\mathrm{Ca}^{2+}$ release mediates tumor progression [29]. Additionally, cell density is increased when tissue stretching results in a sparse cell distribution, which activates Piezo1 and stimulates cell proliferation [30]. These findings indicate that intracellular $\mathrm{Ca}^{2+}$ is associated with the melanoma malignancy.

We have focused on the interaction between the tumour mechanical microenvironment and biochemical signalling pathways. Our present study showed that Piezo1 knockdown inhibited invasion, metastasis, transendothelial migration, metastasis-related genes, including matrix metalloproteinases (MMP2, MMP9), and EMT (N-cadherin and E-cadherin). Next, we treated the cells with either LY294002 (PI3K inhibitor), or Yoda1 (Piezo1 activator) alone or in combination, and found that invasion, metastasis and transendothelial migration were activated and inhibited or partially activated, respectively. Previous studies reported that tumour tissue stiffness provides a mechanical microenvironment to activate Piezo1 and that the expression of genes involved in ECM remodeling can modulate tissue stiffness [9, 31]. Numerous studies have suggested that EMT contributes to invasion and metastasis in melanoma [32, 33]. Moreover, tumour progression is dependent on extracellular matrix remodeling, fibroblast and macrophage activation and EMT [34]. Since EMT is associated with the epigenetic signature of ECM remolding genes [35], we hypothesized that the metastatic process in melanoma cells is associated with Piezo1 regulation of ECM remodeling. A previous study showed that Piezo1 regulated the assembly of focal adhesions, activations of integrin signalling and expression of a number of genes involved in ECM remodeling, further increasing tissue stiffness, which in turn activated Piezo1 and elevated the mechanosensory capacity of tumour cells [9].

After discovering that Piezol1 is an oncogene that affects malignant progression, we aimed to investigate the possible mechanism. Tumour cells respond to mechanical bio-signalling pathways, including AKT/YAP-TAZ/ $\beta$-catenin [36]. Here, we found no difference in several candidates except the AKT pathway. Additionally, either a PI3K/AKT signaling pathway inhibitor or the Yoda1 (Piezo1 activator) or the combination of both reversed these phenomena. From this research, we demonstrated the silencing Piezo1 inhibited PI3K/AKT/mTOR pathway and that Yoda1-induced activation of Piezo1 increased $\mathrm{PISK} / \mathrm{AKT} / \mathrm{mTOR}$ levels. Previous studies have revealed that the activation of AKT/mTOR has a crucial effect on the progression of various cancers $[17,18,25,37]$. At present, some anticancer drugs targeting (PI3K/AKT/mTOR) signalling pathways have been developed and have progressed to various stages of 
clinical trials, and some have proven to be effective [38,39]. Our findings provide a possible therapeutic target for the malignant progression of Piezo-induced melanoma.

\section{Conclusion}

In summary, we investigated the oncogenic role of Piezo1 in melanoma and demonstrated the mechanism which Piezo1 regulates malignant progression of melanoma through the PI3K/AKT/mTOR signalling pathway. The function the Piezo1 mechanosensitive ion channel attracts an immediate clinical focus and make it a target for drug designed against cancer progression.

\section{Abbreviations}

HUVEC: human umbilical vein vascular endothelial cell; PI3K: Phosphatidylinositol 3-kinase; MMPs: Matrix metalloproteinases

\section{Declarations}

\section{Acknowledgements}

Not applicable.

\section{Author contributions}

S.Z. performed most of the experiments, treated data, and wrote the manuscript; S.C. performed animal experiments; M.G.,W.Z.,S.W. and Z.Z. participated in analyzing the data and organized the Figures; L.W., Y.Y. and W.Q. read and reviewed the manuscript. Q.M. provided important support for the design of supplementary experiments; S.W. and Z.W designed the experiment and reviewed the manuscript. All authors read and approved the final manuscript.

\section{Funding}

This study was supported by the National Natural Science Foundation of China (NCFC 82072702/ 81872008); National Key R\& D Projects Sub-project (No.2019YFC1315900); the Science and Technology Innovation as a Whole Plan Projects of Shaanxi Province, China (No. 2016KJZDSF01-05-01).

\section{Availability of data and materials}

All data generated or analyzed during this study are available from the corresponding author on reasonable request.

\section{Conflict of Interest}

The authors declare no conflict of interest. 


\section{Ethics approval and consent to participate}

Studies involving animals was approved by the Ethical Committee of the First Affiliated Hospital of Xi'an Jiaotong University, Xi'an, China.

\section{Consent for publication}

We have obtained consent to publish from the participants to report individual patient data.

\section{References}

1. Siegel RL, Miller KD, Fuchs HE, Jemal A. Cancer Statistics, 2021. Ca-Cancer J Clin. 2021;71:7-33.511

2. Pennino FP, Murakami M, Zollo M, Robertson ES. The metastasis suppressor protein NM23-H1 modulates the PI3K-AKT axis through interaction with the p110alpha catalytic subunit. Oncogenesis. 2021;10:34.553

3. Rodriguez-Hernandez I, Maiques O, Kohlhammer L, Cantelli G, Perdrix-Rosell A, Monger J, et al. WNT11-FZD7-DAAM1 signalling supports tumour initiating abilities and melanoma amoeboid invasion. Nat Commun. 2020;11.525

4. Coste B, Mathur J, Schmidt M, Earley TJ, Ranade S, Petrus MJ, et al. Piezo1 and Piezo2 Are Essential Components of Distinct Mechanically Activated Cation Channels. Science. 2010;330:55-60.514

5. Volkers L, Mechioukhi Y, Coste B. Piezo channels: from structure to function. Pflug Arch Eur J Phy. 2015;467:95-9.515

6. Saotome K, Murthy SE, Kefauver JM, Whitwam T, Patapoutian A, Ward AB. Structure of the mechanically activated ion channel Piezol. Nature. 2018;554:481-+.516

7. Gottlieb PA, Sachs F. Piezo1 Properties of a cation selective mechanical channel. Channels. 2012;6:214-9.517

8. Li J, Hou B, Beech DJ. Endothelial Piezo1: Life depends on it. Channels. 2015;9:1-2.519

9. Chen X, Wanggou S, Bodalia A, Zhu M, Dong WF, Fan JJ, et al. A Feedforward Mechanism Mediated by Mechanosensitive lon Channel PIEZO1 and Tissue Mechanics Promotes Glioma Aggression. Neuron. 2018;100:799-+.521

10. Yu Y, Wu X, Liu S, Zhao H, Li B, Zhao H, et al. Piezo1 regulates migration and invasion of breast cancer cells via modulating cell mechanobiological properties. Acta Biochim Biophys Sin (Shanghai). 2021;53:10-8.522

11. Wang SP, Cao S, Arhatte M, Li DH, Shi Y, Kurz S, et al. Adipocyte Piezo1 mediates obesogenic adipogenesis through the FGF1/FGFR1 signaling pathway in mice. Nat Commun. 2020;11.523

12. Girard CA, Lecacheur M, Ben Jouira R, Berestjuk I, Diazzi S, Prod'homme V, et al. A Feed-Forward Mechanosignaling Loop Confers Resistance to Therapies Targeting the MAPK Pathway in BRAFMutant Melanoma. Cancer Res. 2020;80:1927-41.551 
13. Perotti V, Baldassari P, Molla A, Nicolini G, Bersani I, Grazia G, et al. An actionable axis linking NFATc2 to EZH2 controls the EMT-like program of melanoma cells. Oncogene. 2019;38:4384-96.527

14. Wicklein D, Otto B, Suling A, Elies E, Luers G, Lange T, et al. CEACAM1 promotes melanoma metastasis and is involved in the regulation of the EMT associated gene network in melanoma cells. Sci Rep. 2018;8.528

15. Kessenbrock K, Plaks V, Werb Z. Matrix Metalloproteinases: Regulators of the Tumor Microenvironment. Cell. 2010;141:52-67.529

16. Galban S, Apfelbaum AA, Espinoza C, Heist K, Haley H, Bedi K, et al. A Bifunctional MAPK/PI3K Antagonist for Inhibition of Tumor Growth and Metastasis. Mol Cancer Ther. 2017;16:2340-50.554

17. Yue Y, Hui K, Wu S, Zhang M, Que T, Gu Y, et al. MUC15 inhibits cancer metastasis via PI3K/AKT signaling in renal cell carcinoma. Cell Death Dis. 2020;11:336.556

18. Polivka J, Jr., Janku F. Molecular targets for cancer therapy in the PI3K/AKT/mTOR pathway. Pharmacol Ther. 2014;142:164-75.555

19. Siegel RL, Miller KD, Jemal A. Cancer Statistics, 2017. CA Cancer J Clin. 2017;67:7-30.540

20. Erdmann F, Lortet-Tieulent J, Schuz J, Zeeb H, Greinert R, Breitbart EW, et al. International trends in the incidence of malignant melanoma 1953-2008--are recent generations at higher or lower risk? Int J Cancer. 2013;132:385-400.559

21. Kai F, Laklai H, Weaver VM. Force Matters: Biomechanical Regulation of Cell Invasion and Migration in Disease. Trends in cell biology. 2016;26:486-97.530

22. Kim SE, Coste B, Chadha A, Cook B, Patapoutian A. The role of Drosophila Piezo in mechanical nociception. Nature. 2012;483:209-12.532

23. Li CY, Rezania S, Kammerer S, Sokolowski A, Devaney T, Gorischek A, et al. Piezo1 forms mechanosensitive ion channels in the human MCF-7 breast cancer cell line. Sci Rep. 2015;5.535

24. Zhang J, Zhou Y, Huang T, Wu F, Liu L, Kwan JSH, et al. PIEZO1 functions as a potential oncogene by promoting cell proliferation and migration in gastric carcinogenesis. Mol Carcinog. 2018;57:114455.534

25. Han Y, Liu C, Zhang D, Men H, Huo L, Geng Q, et al. Mechanosensitive ion channel Piezo1 promotes prostate cancer development through the activation of the Akt/mTOR pathway and acceleration of cell cycle. Int J Oncol. 2019;55:629-44.541

26. Lee YH, Lai CW, Cheng YC. Fluid Shear Stress Induces Cell Cycle Arrest in Human Urinary Bladder Transitional Cell Carcinoma Through Bone Morphogenetic Protein Receptor-Smad1/5 Pathway. Cell Mol Bioeng. 2018;11:185-95.536

27. Ueasilamongkol P, Khamphaya T, Guerra MT, Rodrigues MA, Gomes DA, Kong Y, et al. Type 3 Inositol 1,4,5-Trisphosphate Receptor Is Increased and Enhances Malignant Properties in Cholangiocarcinoma. Hepatology. 2020;71:583-99.537

28. Cui C, Merritt R, Fu L, Pan Z. Targeting calcium signaling in cancer therapy. Acta Pharm Sin B. $2017 ; 7: 3-17.538$ 
29. He F, Yu J, Yang J, Wang S, Zhuang A, Shi H, et al. m(6)A RNA hypermethylation-induced BACE2 boosts intracellular calcium release and accelerates tumorigenesis of ocular melanoma. Mol Ther. 2021;29:2121-33.560

30. Gudipaty SA, Lindblom J, Loftus PD, Redd MJ, Edes K, Davey CF, et al. Mechanical stretch triggers rapid epithelial cell division through Piezo1. Nature. 2017;543:118-21.542

31. Retailleau K, Duprat F, Arhatte M, Ranade SS, Peyronnet R, Martins JR, et al. Piezo1 in Smooth Muscle Cells Is Involved in Hypertension-Dependent Arterial Remodeling. Cell Rep. 2015;13:116171.518

32. Pearlman RL, Montes de Oca MK, Pal HC, Afaq F. Potential therapeutic targets of epithelialmesenchymal transition in melanoma. Cancer Lett. 2017;391:125-40.543

33. Huergo-Zapico L, Parodi M, Cantoni C, Lavarello C, Fernandez-Martinez JL, Petretto A, et al. NK-cell Editing Mediates Epithelial-to-Mesenchymal Transition via Phenotypic and Proteomic Changes in Melanoma Cell Lines. Cancer Res. 2018;78:3913-25.544

34. Catalano V, Turdo A, Di Franco S, Dieli F, Todaro M, Stassi G. Tumor and its microenvironment: a synergistic interplay. Semin Cancer Biol. 2013;23:522-32.539

35. Peixoto P, Etcheverry A, Aubry M, Missey A, Lachat C, Perrard J, et al. EMT is associated with an epigenetic signature of ECM remodeling genes. Cell Death Dis. 2019;10:205.545

36. Broders-Bondon F, Nguyen Ho-Bouldoires TH, Fernandez-Sanchez ME, Farge E. Mechanotransduction in tumor progression: The dark side of the force. J Cell Biol. 2018;217:1571-87.550

37. Aksamitiene E, Kiyatkin A, Kholodenko BN. Cross-talk between mitogenic Ras/MAPK and survival PI3K/Akt pathways: a fine balance. Biochem Soc Trans. 2012;40:139-46.547

38. Sabbah DA, Hajjo R, Bardaweel SK, Zhong HA. Phosphatidylinositol 3-kinase (PI3K) inhibitors: a recent update on inhibitor design and clinical trials (2016-2020). Expert Opin Ther Pat. 2021:1-16.561

39. Dey N, De P, Leyland-Jones B. PI3K-AKT-mTOR inhibitors in breast cancers: From tumor cell signaling to clinical trials. Pharmacol Ther. 2017;175:91-106.562

\section{Figures}



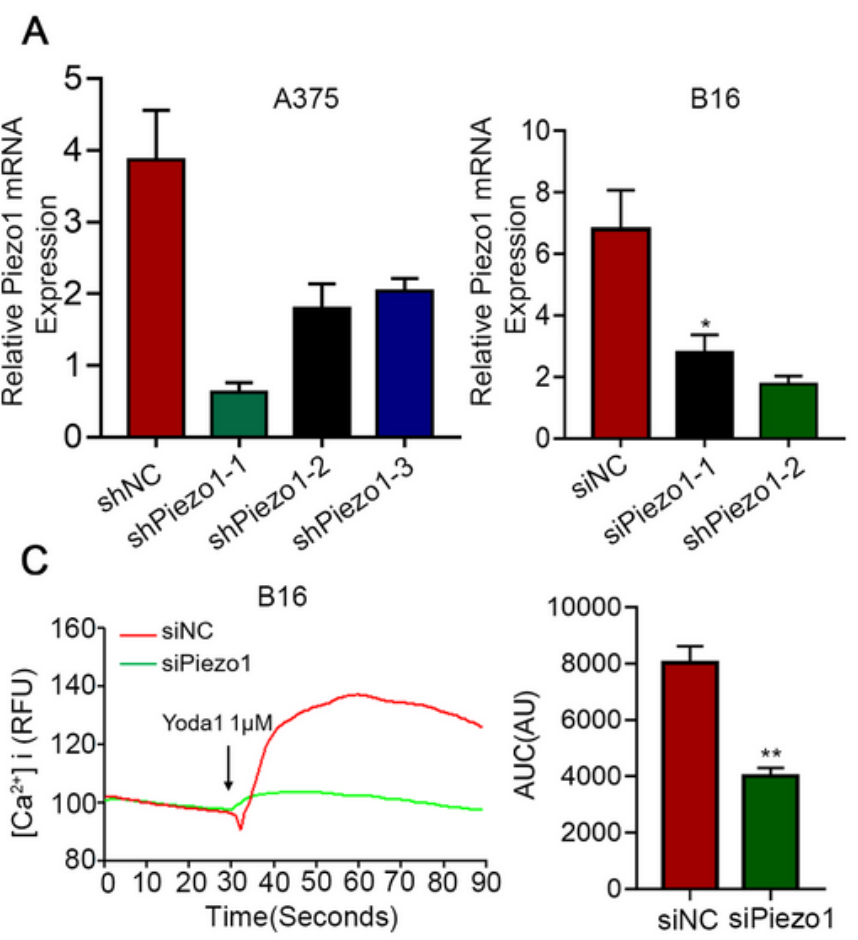

$\mathrm{E}$
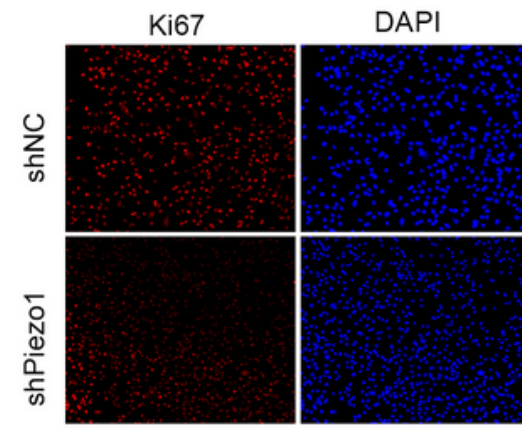

A375

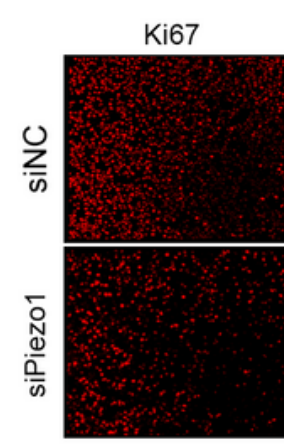

Merge

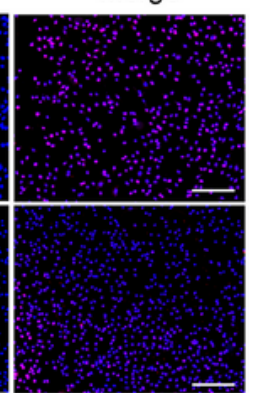

Merge

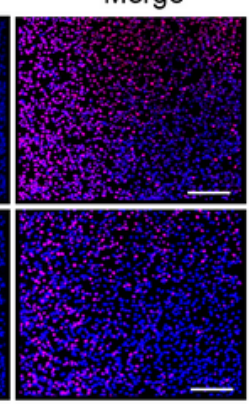

B16

B
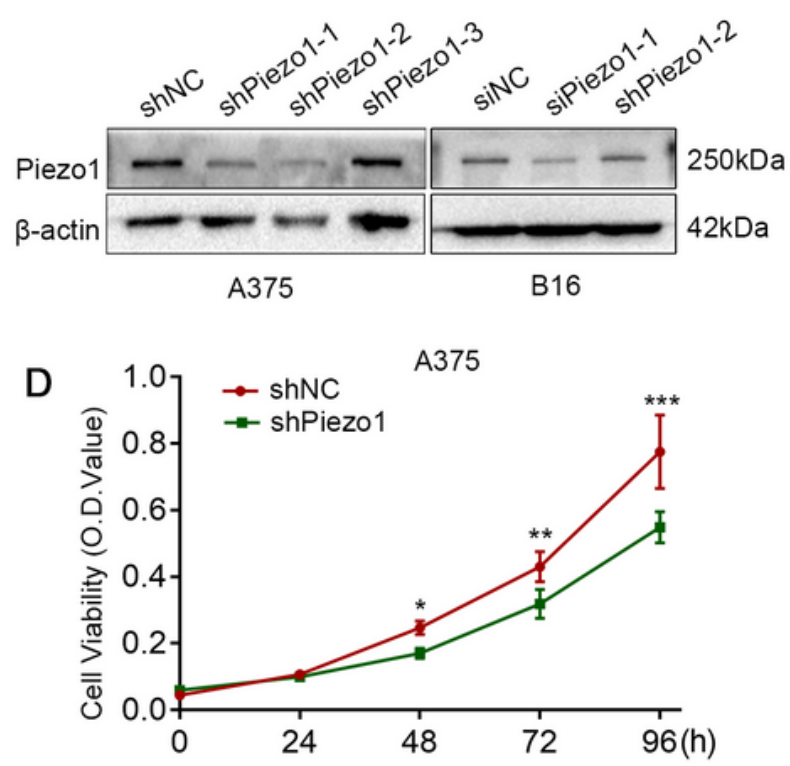

B16
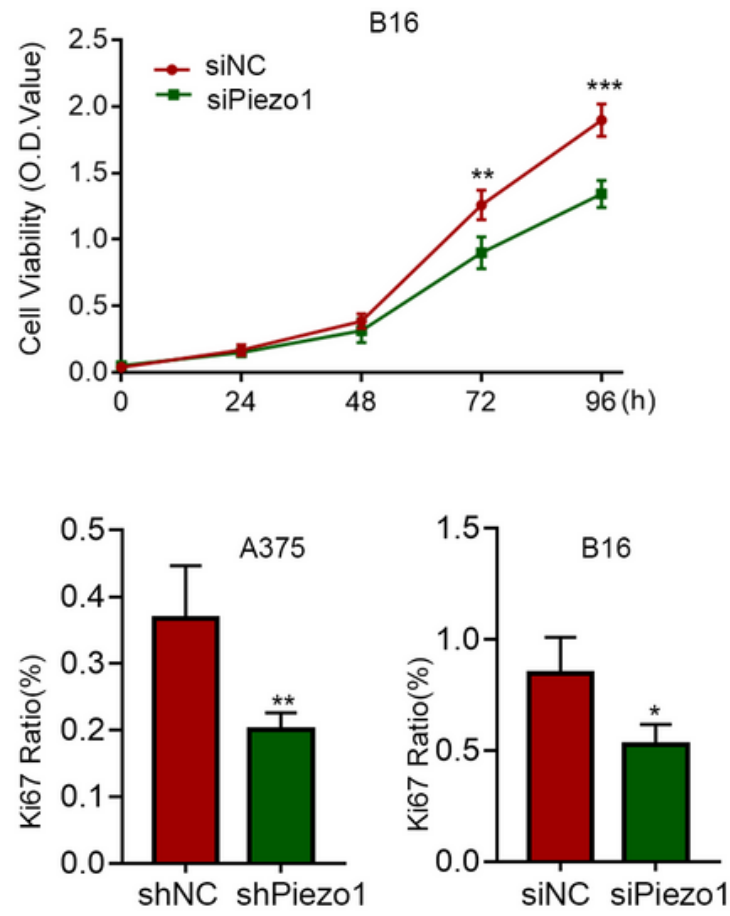

\section{Figure 1}

Piezo1 promotes melanoma cell viability a, b Quantitative real-time PCR and western blotting of Piezo1 mRNA and protein levels in A375, and B16 cells transfected with Piezo1 shRNA/siRNA (shPiezo1/siPiezo1) or negative control (NC). 18S and $\beta$-actin were used as loading controls. c Fluo-4-AM B16 cells (shNC, $n=30$; shPiezo1, $n=36$ ) were exposed to $10 \mu \mathrm{M}$ Yoda1 and [Ca2+]i was determined as fluorescence intensity (RFU, relative fluorescence units); Bar diagrams show the area under the curve 
(AUC) of the Ca2+-transient. d Cell viability was detected by CCK8 assay with treatment as indicated. e An Ki67 incorporation assay for staining proliferating cells (red indicates Ki67-incorporated cells, blue indicates nuclear staining with DAPI) was conducted in A375 and B16 cells; Bar diagrams showing the average of Ki67 to DAPI ratio in Piezo1 knockdown group cells and control group cells presented as the mean $\pm \operatorname{SEM}(n=7)$. Scale bars, $100 \mu \mathrm{m}$. Shown are mean values \pm SEM.; ${ }^{*} p<0.05 ;{ }^{* \star} p<0.01 ; * \star \star p<$ 0.001 .

A
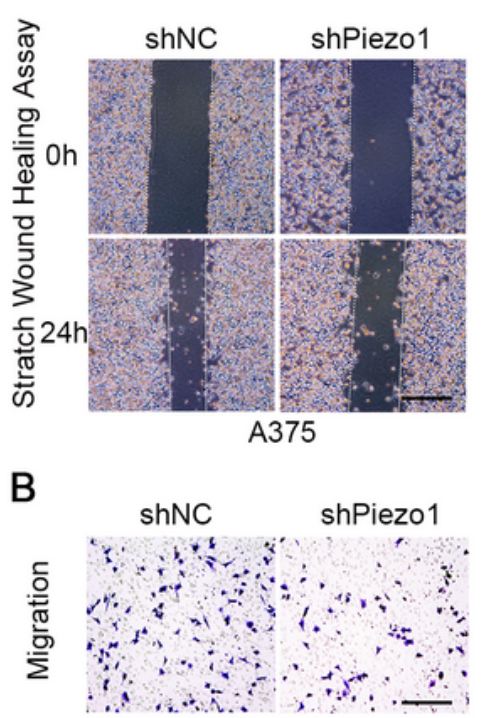

A375

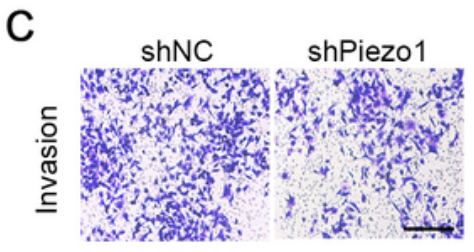

A375

D
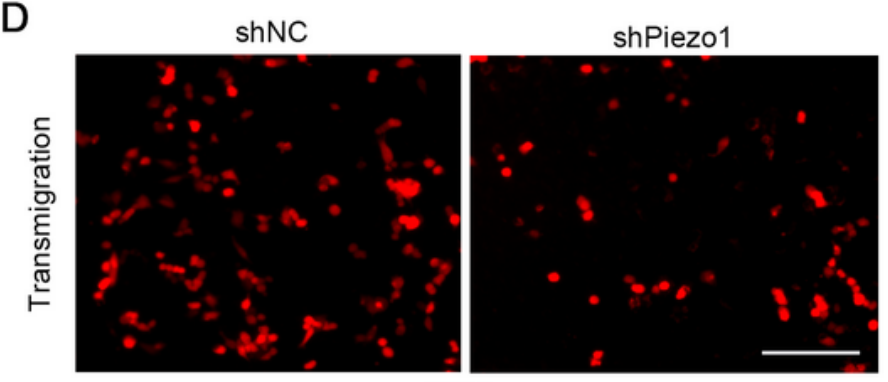

A375

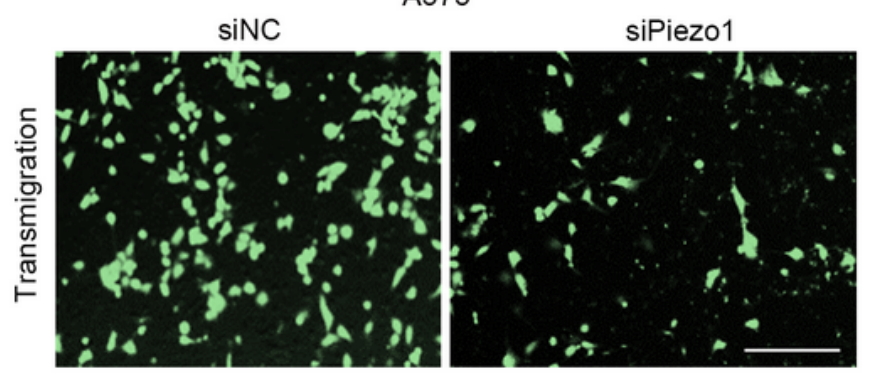

B16

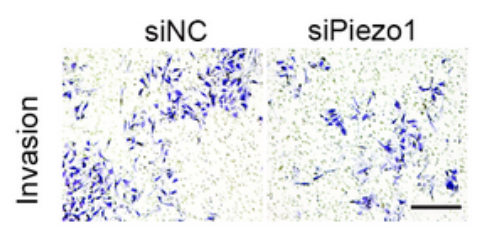

B16
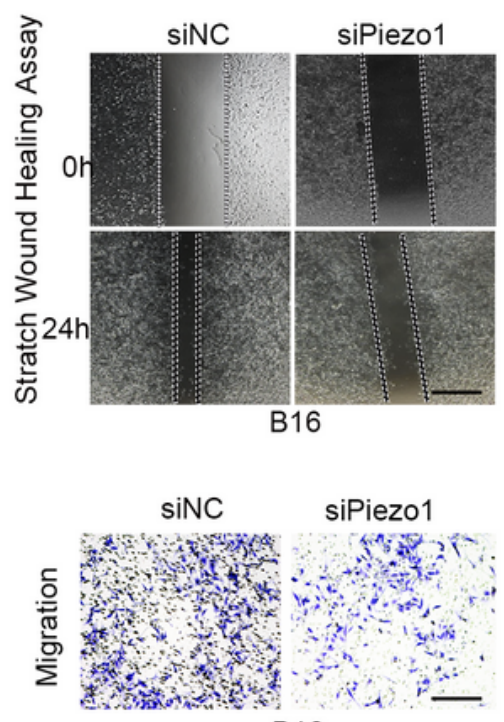

B16
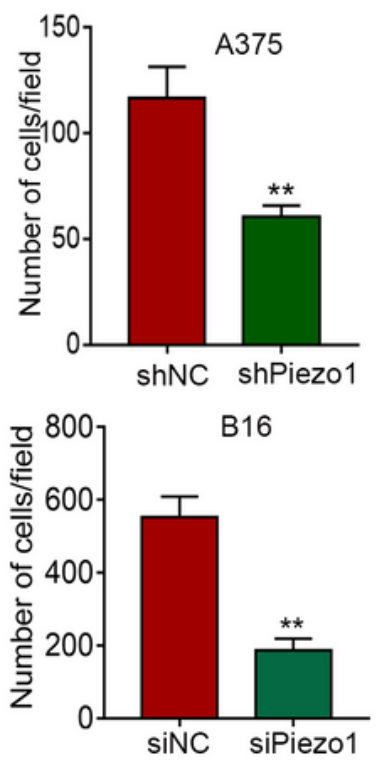
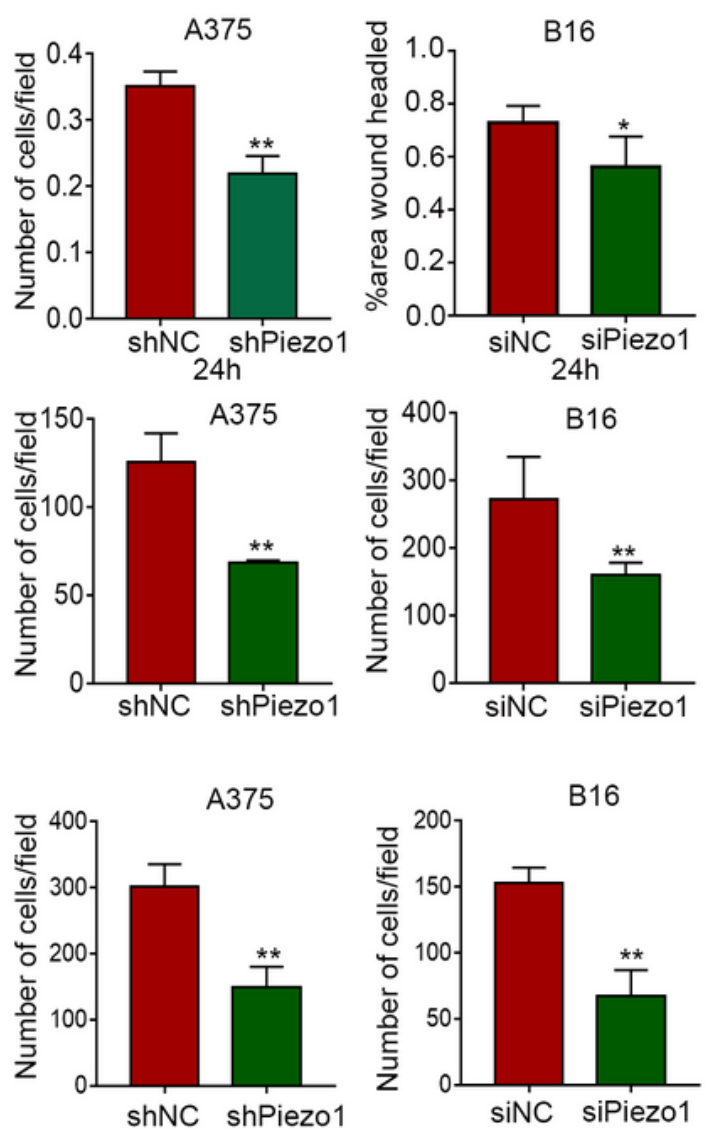

B16

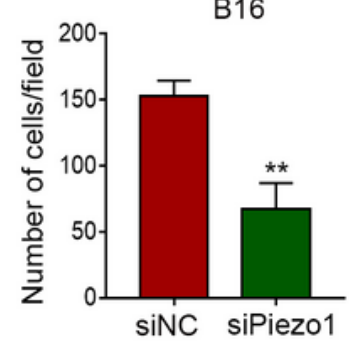

siNC siPiezo1

Figure 2 
Knockdown of Piezo1 inhibits the migration, invasion and transendothelial migration of melanoma cells in vitro. $a, b$ Migration capacity was assessed by the wound healing assay and transwell assay in A375 and B16 cells. Quantification data showed on the right. Scale bars, $100 \mu \mathrm{m}$ c Invasion capacity was assessed by the transwell assay with Matrigel in A375 and B16 cells. Quantitative data showed on the right. Scale bars, $100 \mu \mathrm{m}$. $d$ Transendothelial migration ability was assessed by the transwell assay in A375and B16 cells. Quantitative data showed on the right. Scale bars, $50 \mu \mathrm{m}$. Shown are mean values \pm SEM.; ${ }^{*}<<0.05 ; * \star p<0.01 ; * * * p<0.001$.
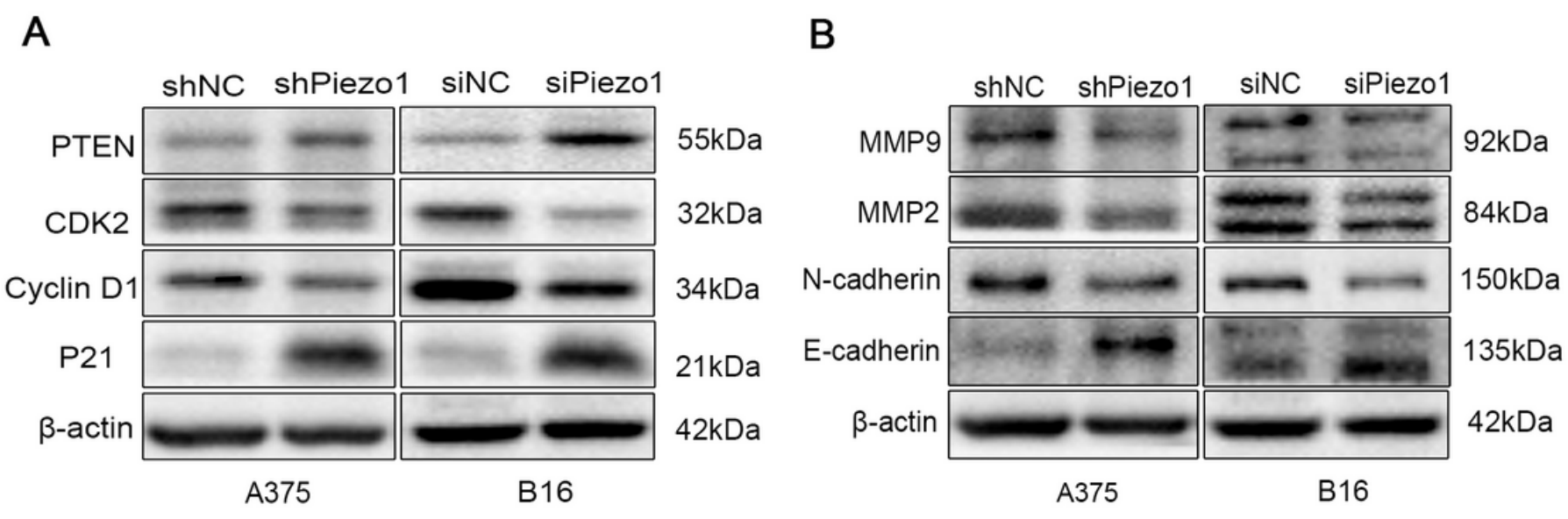

C

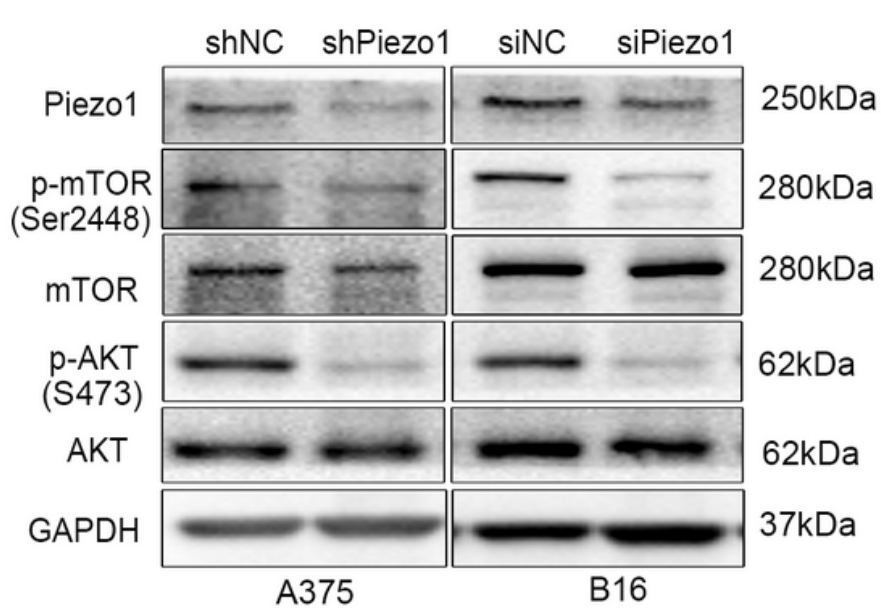

D

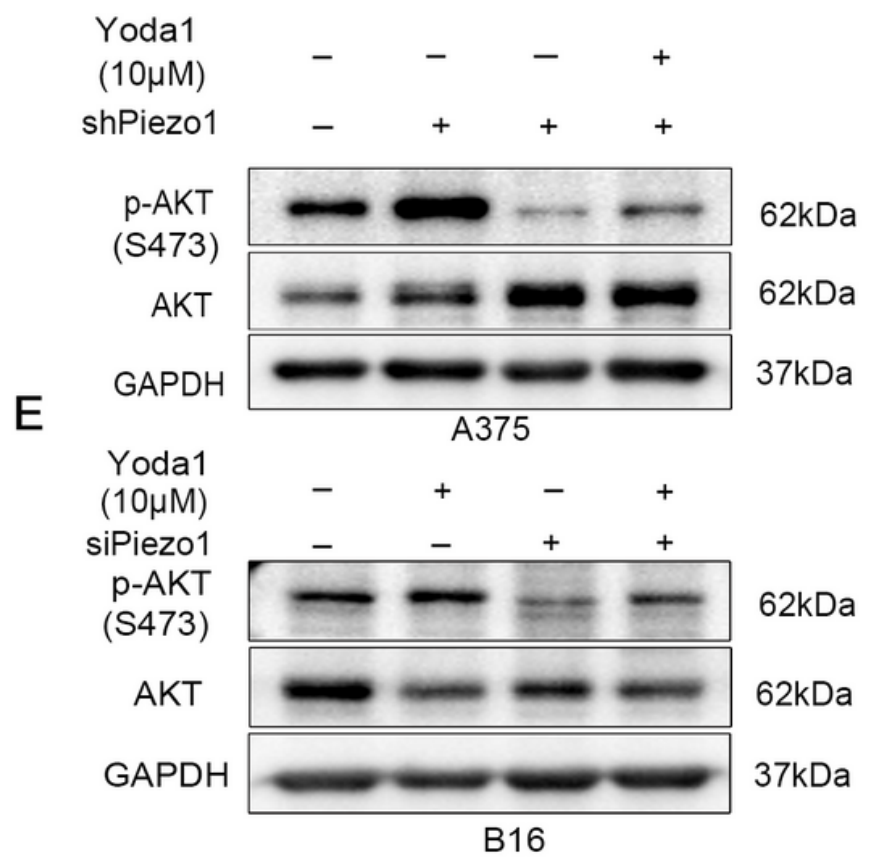

\section{Figure 3}

Piezo1 inhibition blocks invasion-, metastasis- and cell cycle- related genes in vitro. a Cell cycle-related proteins were detected by western blotting using the indicated antibodies. The images show the changes in the proteins PTEN, P21, Cyclin D1, and CDK2 in A375 and B16 cells. $\beta$-actin was used as a loading control. b The gene expression of E-cadherin, N-cadherin, MMP2 and MMP9 were detected by a western 
blot assay in A375 and B16 cells after Piezo1 knockdown. $\beta$-actin served as an internal control. Piezo1 inhibition inactivates the PI3k/AKT pathway c Western blotting analysis of total and phosphorylated AKT, and mTOR in A375 and B16 cells after Piezo1 knockdown. GAPDH served as loading control. d Western blotting analysis of total and phosphorylated AKT, mTOR in A375 and B16 cells transfected with $\mathrm{sh} / \mathrm{siPiezo1}$ or sh/siNC after treatment with Yoda1 (a Piezo1 activator) for $24 \mathrm{~h}$. GAPDH was used as a loading control.

A

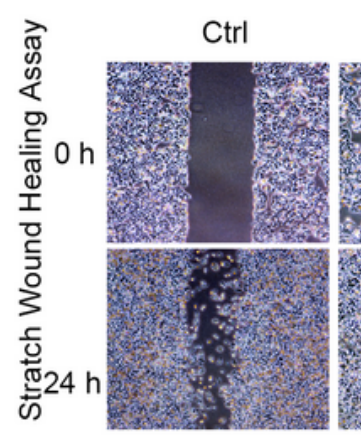

B

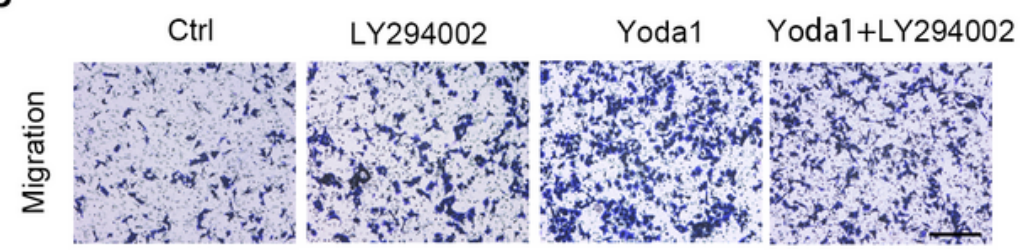

C

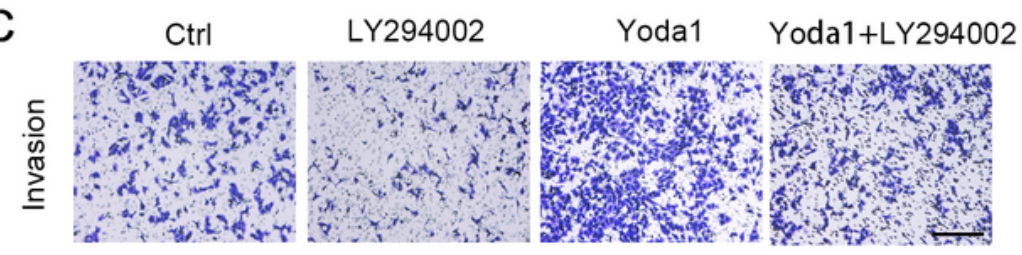

D

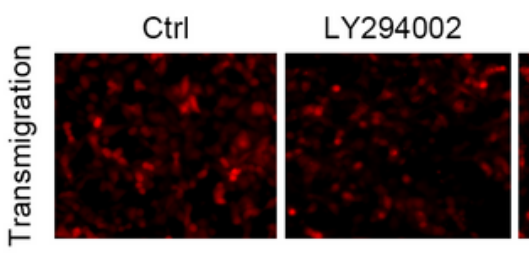

E

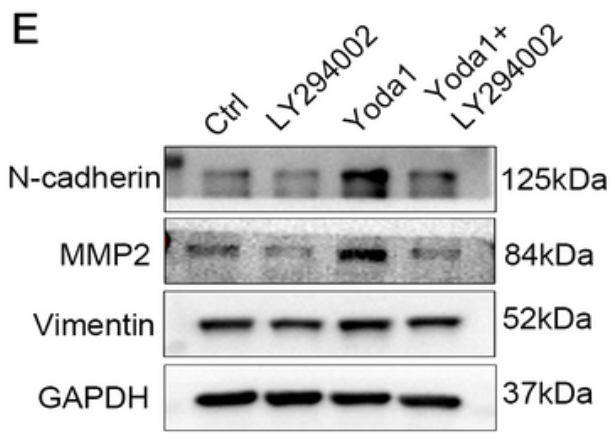

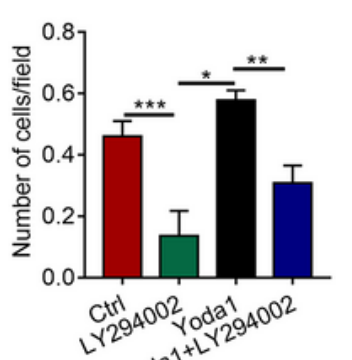
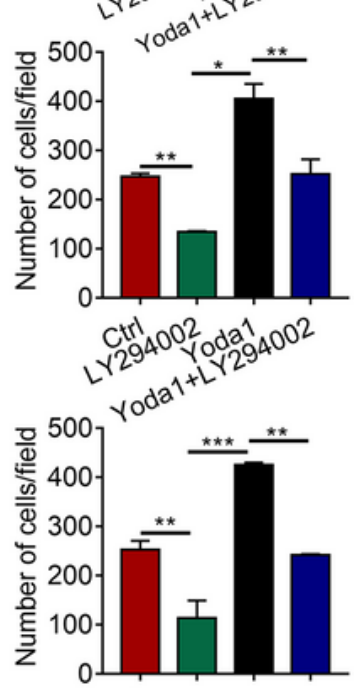

${ }_{4}{ }^{2+1} 400^{2}{ }_{1}^{2} a_{40}$

Lre

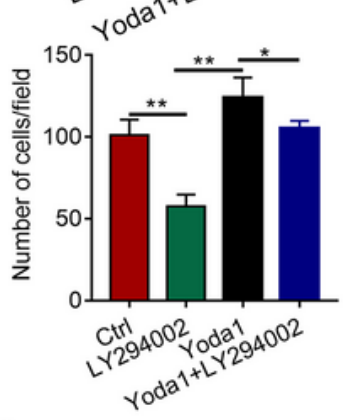

$\mathrm{F}$

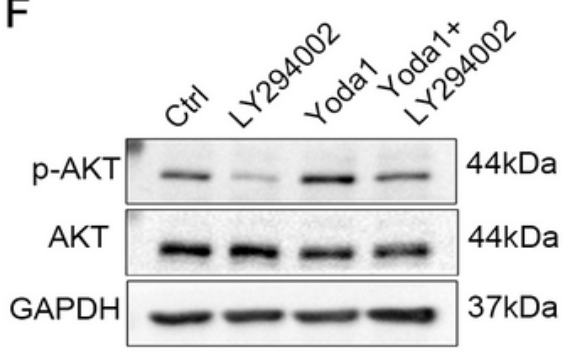


Piezo1 promotes melanoma cell proliferation, metastasis and invasion through the PI3K/AKT signalling pathway. a Representative images of wound healing in A375 cells treated with PI3K inhibitor LY294002, Piezo1 activator Yoda1 and both. Quantification analysis was shown on the right $(\mathrm{N}=5)$. The scale bars, $100 \mu \mathrm{m} . \mathrm{b}, \mathrm{c}, \mathrm{d}$ Representative pictures of transwell migration, invasion and transmigration in A375 cells treated with PI3K inhibitor LY294002, Piezo1 activator Yoda1 and both. Quantification analysis was shown on the right $(\mathrm{N}=3)$. e Western blot analysis of of MMP2, N-cadherin, Vimentin and PI3K/AKT signaling in A375 cells treated with PI3K inhibitor LY294002, Piezo1 activator Yoda1 and both. GAPDH was used as a loading control $(N=3)$. The scale bars, $100 \mu \mathrm{m}$. ( ${ }^{\star} P<0.05,{ }^{\star *} P<0.01$, $\left.{ }^{\star \star *} P<0.001\right)$ 
A

shNC

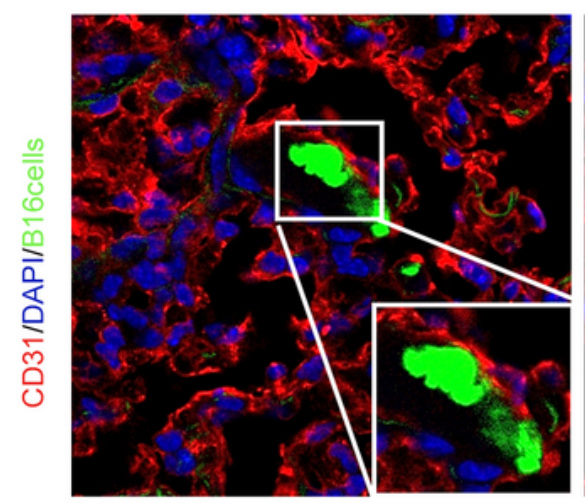

shPiezo1

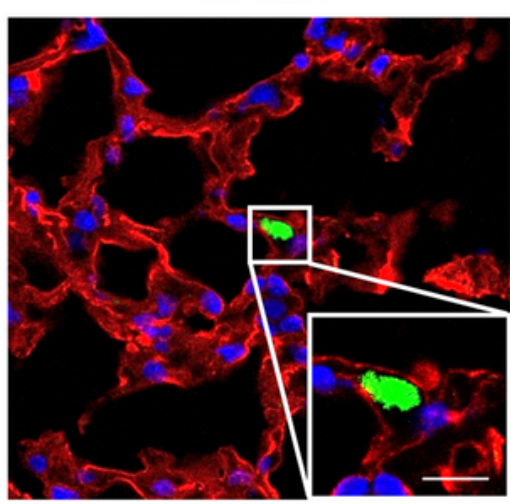

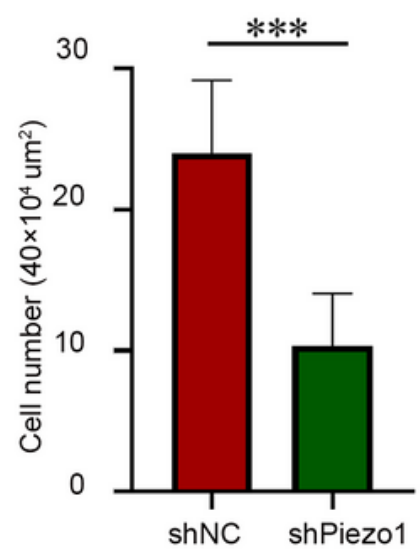

B
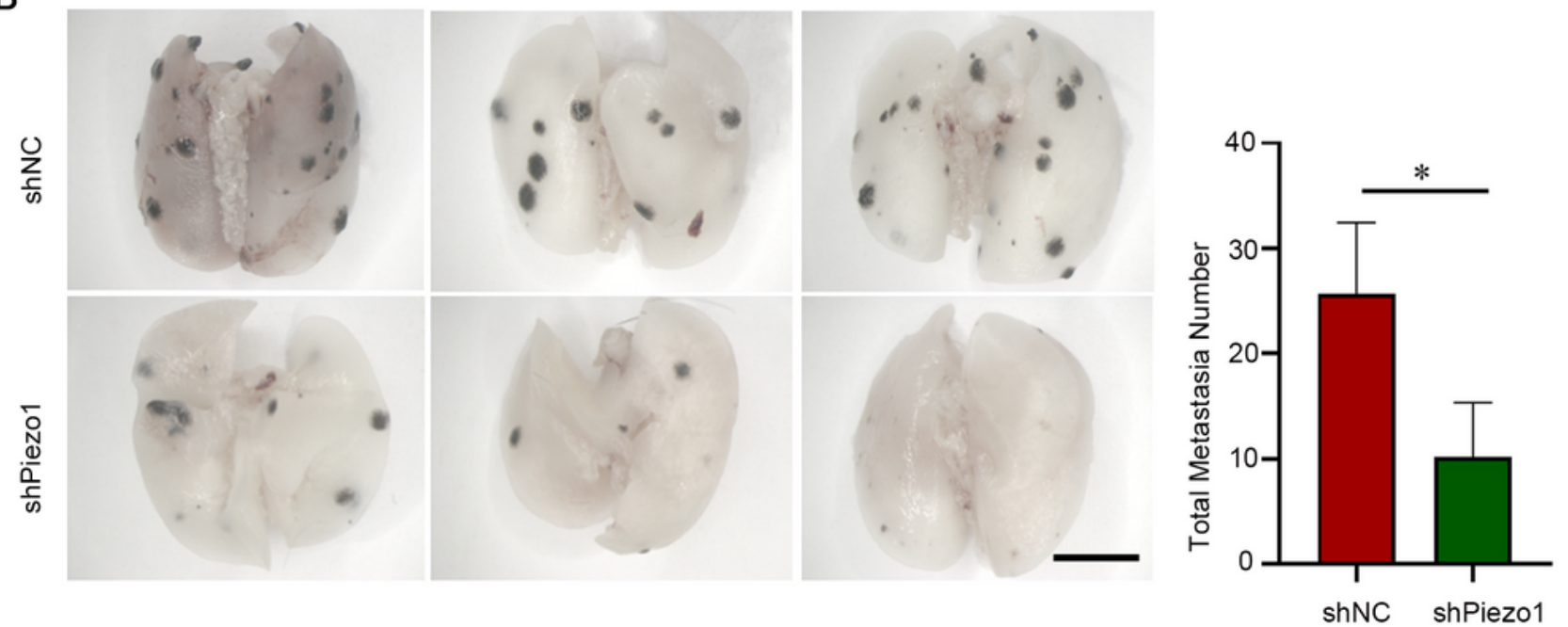

C

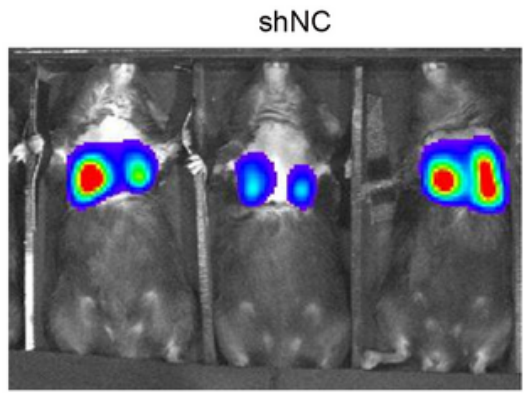

shPiezo1

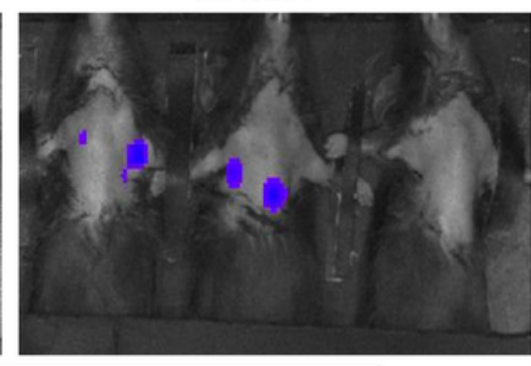

Tail vain injection model

Radiance (Photons $\mathrm{s}^{-1} \mathrm{~cm}^{2} \mathrm{sr}^{-1}$ ) Colour scale Min $0.5 \times 10^{4}$

Max $5.96 \times 10^{4}$
Luminescence

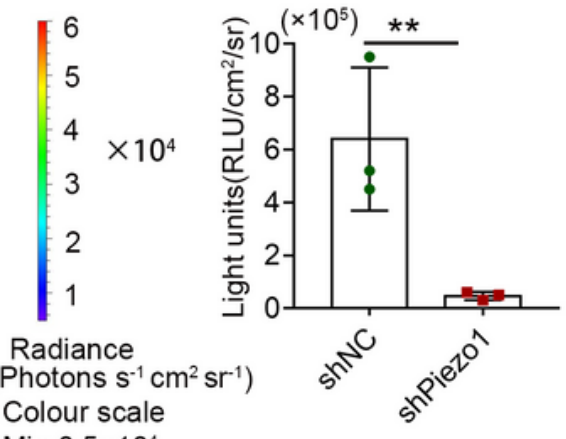

\section{Figure 5}

Knockdown of Piezo1 attenuated melanoma metastasis and transendothelial migration in vivo. a Immunofluorescence respectively after injecting B16 cells (shPiezo1and shNC fluorescently labelled) into mice for 6 hours (red indicated the blood vessel labeled with CD31, green indicated the cell with GFP, and blue indicated nucleus). Bar graph showed the number of cells extravasated into the vasculature. The scale bars, $20 \mu \mathrm{m}$. b The number of lung surface metastatic lesions from the shPiezo1 and shNC groups 
was calculated. Bar graph showing the average of the number of lung surface metastatic lesions. The scale bars, $10 \mathrm{~mm}$. c Representative bioluminescence images from the shPiezo1 $(n=10)$ and shNC $(n=9)$ groups are shown in the left panel. The scale shows the level of signal strength. Shown are mean values \pm SEM.; ${ }^{*} p<0.05 ;{ }^{* *} p<0.01 ; * * \star p<0.001$.

\section{Supplementary Files}

This is a list of supplementary files associated with this preprint. Click to download.

- supplementary2.tif

- supplementary1.tif 